\section{Paciente con Alzheimer en fase moderada y su familia: análisis bibliográfico de un caso clínico}

\section{Patient with Alzheimer in moderate phase and his family: bibliographic analysis of a clinical case analysis of a clinical case}

\begin{abstract}
Alicia Lizarraga-Cía'
Mónica Vázquez-Calatayud ${ }^{2, *}$

1. Estudiante de Grado en Enfermería. Universidad de Navarra. Pamplona. Navarra. España.

2. Doctora en Ciencias de la Enfermería. Profesor Colaborador, Facultad de Enfermería, Universidad de Navarra. Grupo de investigación ICCP-UNAV, Innovación para un Cuidado Centrado en la Persona. IdisNA, Instituto de Investigación Sanitaria de Navarra, Pamplona, Navarra, España.
\end{abstract}

${ }^{*}$ Autor para correspondencia.

Correo electrónico: mvazca@unav.es (Mónica Vázquez-Calatayud).

Recibido el 21 de abril de 2020; aceptado el 16 de junio de 2020.

\section{RESUMEN}

Presentación del caso: Paciente de 76 años residente en un centro geriátrico, con Alzheimer en fase moderada, que manifiesta agitación

y estrés ante una situación incómoda. Ante la aparición de estos cambios conductuales, tanto la enfermera a su cuidado como la familia desconocen cómo actuar. Objetivos: Identificar, con base en la literatura científica, las intervenciones de enfermería más efectivas para manejar y prevenir cambios conductuales en una persona con Alzheimer en fase moderada y valorar la integración familiar en dichas estrategias de cuidado. Revisión bibliográfica: Se realizó una búsqueda bibliográfica en las bases de datos CINAHL y PubMed, seleccionándose 7 artículos.

Resultados: Se han identificado cuatro tipos de intervenciones de enfermería para el manejo y prevención de cambios conductuales: sensitivas, ambientales, físicas y psicoemocionales. Existen escasas intervenciones desarrolladas en centros geriátricos que integren a la familia en el cuidado de los pacientes. Conclusión: La ejecución de las intervenciones de enfermería seleccionadas podrá favorecer una mejoría tanto en el manejo como en la prevención de los cambios conductuales.

La realización de dichas actividades por parte de la unidad familiar incrementa los efectos positivos en la familia, la residente y en el equipo de enfermería. Sin embargo, se precisa un número mayor de estudios que integren a la familia para poder generalizar los resultados.

PALABRAS CLAVE: Alzheimer, cambios conductuales, enfermería, familia, intervención.

\section{ABSTRACT}

Case presentation: 76-year-old patient living in a geriatric centre with moderate-phase Alzheimer, who shows agitation and stress in an uncomfortable situation. Faced with the appearance of these behavioural changes, both the nurse in charge and the family do not know how to act. Objectives: To identify the most effective nursing interventions to manage and prevent behavioural changes in a person with Alzheimer's in a moderate phase, and assess family integration into these care strategies. Literature review: A bibliographic search was carried out on the CINAHL and PubMed databases, selecting 7 articles. Results: Four types of nursing interventions have been identified for the management and prevention of behavioural changes: sensory, environmental, physical and psycho-emotional. There are few interventions developed in geriatric centres that integrate the family in the care of patients. Conclusion: The implementation of the selected nursing interventions creates an improvement in both the management and prevention of behavioural changes. The implementation of such activities by the family unit increases the positive effects on the family, the resident and the nursing team. However, more studies that integrate the family are needed to generalize the results.

KEYWORDS: Alzheimer, family, intervention, nursing, wandering behaviour.

\section{- INTRODUCCIÓN}

El envejecimiento de la población ha supuesto un incremento de las enfermedades degenerativas. La enfermedad de Alzheimer (EA) es la más frecuente y representa a nivel mundial entre el $60 \%$ y el $70 \%$ de las demencias ${ }^{1}$. La EA es "una dolencia degenerativa cerebral primaria, de etiología desconocida, que presenta síntomas neuropáticos y neuroquímicos característicos"'.

La EA constituye uno de los principales retos a nivel mundial. Se estima que 50 millones de personas de todo el mundo viven con esta demencia desde 2018. Esta cifra aumentará más del triple, hasta los 152 millones, para el ańo $2050^{2}$. En Espańa, 800000 personas están afectadas por esta enfermedad y se manifiestan más de 40000 casos nue- vos al año, siendo la segunda causa de muerte más frecuente ${ }^{3}$. A nivel nacional, el coste por paciente de Alzheimer se estima que ronda entre 27000 y 37000 euros anuales ${ }^{3}$.

Dado que esta enfermedad va deteriorando de forma progresiva todas las capacidades cognitivas e intelectuales de la persona, conforme avanza se producen alteraciones conductuales que se manifiestan de formas diferentes: agresividad (verbal y física), negatividad, inquietud, agitación, deambulación errática, cambios de apetito o alteraciones del sueño $0^{4}$. Es fundamental para el cuidado de los pacientes con EA saber en qué fase se encuentran de la enfermedad, debido a que, según la fase, el tratamiento y los resultados esperados serán diferentes 5 . Los pacientes con cambios conductuales y psicológicos no tratados tienen una progresión de la enfermedad más rápida ${ }^{6}$. 
Estos cambios de comportamiento se producen en la fase moderada de la enfermedad e impactan de manera notable tanto en la calidad de vida de la persona afectada como en la de sus familiares cuidadores, lo que implica que el $85 \%$ de estos enfermos sean institucionalizados. En este contexto, el papel de los profesionales sanitarios, y en especial el de enfermería, es clave por su liderazgo y posición cercana ${ }^{7,8}$.

La familia cuidadora de un paciente en fase moderada encuentra mayores dificultades asociadas a las actividades realizadas para el cuidado de ellos en relación con las familias de pacientes en fase inicial ${ }^{9}$. En este sentido, y teniendo en cuenta que los familiares son las personas que con más frecuencia asumen el cuidado de un enfermo con Alzheimer ${ }^{8}$, es importante focalizar la atención tanto en las personas afectadas como en los cuidadores.

La alta prevalencia de la EA, su gran impacto socioeconómico y la complejidad en su abordaje conllevan que sea clave conocer las intervenciones de enfermería más adecuadas para el cuidado de los pacientes con EA y sus familiares. Para ello, se ha llevado a cabo este trabajo con los siguientes objetivos:

1. Describir y contextualizar el caso clínico de un paciente con EA en fase moderada y su familia.

2. Analizar un caso clínico, según la literatura, de un paciente con EA y su familia en la fase moderada de la enfermedad.

\section{- PRESENTACIÓN DEL CASO}

Paciente mujer de 76 años que ingresó hace 5 años en una de las tres plantas de un centro residencial público de la tercera edad situado en el norte de España, a causa de su EA en fase moderada. Dada su larga estancia, era conocida por todo el personal sanitario. En dicha planta había en total 60 residentes dependientes, la mayoría por demencias, accidentes cerebrovasculares y parálisis. Para su atención, la plantilla de enfermería estaba dotada con 2 enfermeras y 8 auxiliares en los turnos de día, 1 enfermera y 8 auxiliares en el turno de tarde y 1 enfermera y 2 auxiliares en el turno de noche.

Esta residente, de etnia gitana, estaba viuda desde hace 8 años y era la matriarca de una familia numerosa. Su núcleo familiar parecía unido, en tanto que la mayor parte del día estaba acompañada por sus 4 hijos, 3 hijas y 10 nietos, quienes se turnaban para visitarla. Su rol en la familia pudo favorecer su carácter autoritario y tendencia a emitir órdenes a las personas de su alrededor. A pesar de ello, la relación con su familia era buena. Sus hijos estaban informados acerca de su enfermedad y tratamiento y velaban en todo momento por su bienestar. Este hecho, en ocasiones, los llevaba a discutir con el personal sanitario por considerar que no se le estaba proporcionando un trato adecuado.

En general, su limitada movilidad obligaba a desplazarla en silla de ruedas. Esta limitación, unida a su falta de colaboración, ya que con frecuencia se agitaba o se oponía debido al proceso de su enfermedad, hacía que para las comidas se priorizara su movilización frente al resto de residentes. A pesar de su resistencia inicial para el traslado, una vez ubicada en su asiento, generalmente se quedaba tranquila, comía adecuadamente y se comportaba de manera correcta.

$\mathrm{Al}$ mes de mis prácticas, en el turno de tarde, ocurrió un incidente con la paciente del caso. En este día concreto, una enfermera de nueva incorporación en el servicio estaba a cargo de esta residente, junto con 9 pacientes más. Era su primer día de trabajo, por lo que intentó apresurarse en su plan de cuidados y se dirigió a los residentes de modo autoritario, ordenándoles aquello que tenían que hacer, como ir a comer o ir al bańo. A la hora de la cena de ese mismo día, intuyendo que esta residente le llevaría más trabajo, ya que así se lo habían transmitido sus compañeras, decidió dejarla para el final para así no perder tiempo.

De este modo, primero, con la ayuda de una auxiliar de enfermería, colocó a todos los residentes a su cargo en su asiento, y después fue a ayudar a la mujer a sentarse en su sitio. Al llegar a ella, esta no colaboró y se negó. La enfermera, nerviosa, comenzó a alzar el tono de voz y con torpeza la sujetó del brazo con fuerza para intentar moverla. Ante este comportamiento, la residente, como era habitual cuando estaba en una situación incómoda, empezó a agitarse, a gritar, a tirar todo lo que encontraba a su alcance, y llegó a golpear a la enfermera para escapar de esa situación. La enfermera solicitó más ayuda a los demás sanitarios, debido a que se sintió impotente y no sabía cómo actuar en esa situación. Acudieron en su ayuda, una enfermera, 2 auxiliares de enfermería y la hija de la paciente. La residente, al sentirse más acorralada, se agitó todavía más. En ese momento, la hija empezó a alterarse también, ya que no entendía el comportamiento de su madre y comenzó a gritarle por ello. Ante esta situación, la enfermera aconsejó a la hija que se distanciara hasta que su madre estuviera más relajada. Después de aplicar varias técnicas como relajación, masajes, tono suave y tranquilidad en el ambiente, el personal de enfermería consiguió que la residente se tranquilizase.

\section{- ANÁLISIS DEL CASO}

\section{Objetivos}

Para analizar el caso descrito se definieron los siguientes objetivos:

1. Identificar, en función de la literatura, cuáles son las intervenciones de enfermería más efectivas para ayudar a prevenir o manejar los cambios conductuales que padecen los enfermos de Alzheimer en la fase moderada de su enfermedad durante su proceso de institucionalización en un centro gerontológico.

2. Contemplar la integración de la unidad familiar en las estrategias de cuidado de los pacientes con EA.

\section{Revisión bibliográfica}

Para responder a los objetivos anteriores, se llevó a cabo una revisión bibliográfica de estudios publicados en las principales bases de datos: PubMed y CINHAL. En estas búsquedas electrónicas, como se ilustra en la figura 1, se combinaron los términos: "Alzheimer", "cambios conductuales", "enfermería", "intervención” y sus sinónimos con los operadores booleanos "AND” y "OR". Para conseguir una mejora de la especificidad de la búsqueda y eludir omitir estudios con relevancia en el caso, se utilizaron los términos $\mathrm{MeSH}$ y las palabras clave identificadas en los estudios seleccionados. En las búsquedas se aplicó como límite el idioma de publicación (inglés y/o español), la fecha de publicación de los estudios (10 últimos años, para garantizar que la bibliografía fuera actual) y la edad (personas mayores de 65 años, debido a que la incidencia es mayor). La selección de los estudios se realizó basdándose en la aplicación de los siguientes criterios de inclusión:

1. Estudios que incluyan intervenciones de enfermería para el cuidado del paciente con EA en fase moderada.

2. Estudios originales y revisiones sobre el tema, siempre y cuando los estudios revisados no estuvieran incluidos en esta revisión.

3. Estudios realizados en el contexto clínico, en el centro residencial. 


Términos de búsqueda
1) Alzheimer*
2) Behavioral symptoms OR wandering behavioral
3) Nurs*
4) Intervention
Alzheimer* AND Behavioral symptoms OR wandering behavioral AND Nurs* AND Intervention
Términos de búsqueda

1) AND 2) AND 3) AND 4)

Términos de búsqueda

Términos de búsqueda

1) AND 2) AND 3) AND 4)

Términos de búsqueda

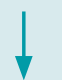

$\downarrow$

\begin{tabular}{|l|l|}
\hline PubMed & \\
\hline 164916 \\
\hline 393598 \\
\hline 945523 \\
\hline 246355
\end{tabular}

742

Introducción de límites: últimos

10 años, idioma inglés y/o español, adultos

PubMed

75

CINAHL

248

Tras lectura del título de los artículos, con su resumen

PubMed

3 CINAHL

Teniendo en cuenta los criterios de inclusión PubMed CINAHL

Figura 1. Estrategia de búsqueda empleada en las bases de datos electrónicas.

\section{Resultados de la búsqueda}

Como resultado de la búsqueda realizada, se localizaron inicialmente 1696 referencias. Tras aplicar los límites de la búsqueda y la lectura de sus títulos se retuvieron 323 artículos. Una vez leído el resumen de dichos artículos se seleccionaron 11. Estos artículos se analizaron en profundidad, descartando cuatro de ellos por no cumplir los criterios de inclusión. Finalmente, los artículos seleccionados para el estudio fueron 7. En la tabla 1 se presentan las características de los estudios seleccionados para esta revisión.

\section{Resultados del análisis del caso}

Basándose en el análisis de los estudios revisados, y en respuesta a los objetivos previamente planteados, se han identificado dos temas principales que han ayudado a analizar el caso de un paciente con EA en fase moderada con cambios conductuales y su familia cuidadora durante su institucionalización en un centro gerontológico.

INTERVENCIONES DE ENFERMERÍA EN EL MANEJO Y PREVENCIÓN DE CAMBIOS CONDUCTUALES EN LA PERSONA CON EA EN FASE MODERADA

Con base en los siete estudios seleccionados se han identificado cuatro tipos de intervenciones, tal y como se resume en la tabla 2: sensitivas, ambientales, físicas y psicoemocionales ${ }^{10-16}$.

\section{Intervenciones sensitivas}

La musicoterapia es una de las intervenciones más utilizadas para el manejo del paciente con EA. En el estudio de Onieva et al. ${ }^{10}$, además de la musicoterapia integran la terapia de reminiscencia (TR) y las técnicas de orientación a la realidad (OR). La TR consiste en una serie intervenciones de enfermería basadas en rememorar eventos, sentimientos y experiencias pasadas con el objetivo de aumentar la adaptación a la evolución de los años. Por otro lado, las técnicas de OR están dirigidas a personas con demencia e implican la repetición y el uso de información relacionada con el tiempo presente, el lugar y las personas en un entorno familiar. En este sentido, Onieva et al. ${ }^{10}$ evidenciaron una reducción de los síntomas de depresión en los pacientes diagnosticados de EA en fase moderada después de la realización de esta intervención sensitiva integrada, 2 veces por semana durante 8 semanas consecutivas. Este resultado indica que el acompańamiento de la música con OR y RT es más efectiva que las intervenciones musicales convencionales, que para producir una mejoría en los pacientes con EA precisan realizarse con mayor frecuencia y en un plazo más largo. Estas intervenciones las realiza el equipo de enfermería, lo que permite que la terapia con música, la TR y la OR estén realmente integradas en las actividades diarias de las personas con $\mathrm{EA}^{10}$. Se considera que estas intervenciones podrían ser beneficiosas para la residente de este caso, ya que, al reducir los síntomas de depresión en un menor tiempo y favorecer su adaptación al medio, podrían favorecer un control en su conducta ante situaciones incómodas. Otra intervención es el programa de terapia ergonómica basada en el comportamiento (TEBC). El objetivo a largo plazo de este programa TEBC es reducir el estrés cognitivo y los cambios conductuales, como la agitación en los residentes con EA en fase moderada. Este programa incluye la musicoterapia y la videoterapia que utilizan reproductores y una biblioteca de DVD ${ }^{11}$. Como se pudo ver en el presente caso, los cambios conductuales que primaban fueron los relacionados con agitación, agobio y estrés. Teniendo esto en cuenta, esta terapia podría también considerarse para paliar estos sentimientos y conseguir prevenir futuros cambios conductuales.

La terapia con masajes es otra de las intervenciones identificada en la literatura; esta impacta de manera positiva tanto en estos pacientes como en su familia. En concreto, los masajes de cabeza y cara pueden prevenir la inquietud, reducir la agitación, el estrés y el dolor en las personas mayores con EA en fase moderada ${ }^{12}$. Esta terapia, además de facilitar el manejo de los cambios conductuales que padecen estos pacientes, 
Tabla 1. Características de los estudios seleccionados en esta revisión

\begin{tabular}{|c|c|c|c|c|c|}
\hline Estudio & Objetivo & Diseño & Muestra & Principales resultados & Limitaciones \\
\hline $\begin{array}{l}\text { Onieva et al. } .^{10} \\
(2018)\end{array}$ & $\begin{array}{l}\text { Explorar el efecto de un programa de música de } \\
8 \text { semanas acompañado de TR y OR sobre los } \\
\text { síntomas de depresión y ansiedad en personas } \\
\text { mayores con EA leve y moderada que viven en una } \\
\text { residencia }\end{array}$ & Estudio piloto & $N=19$ & $\begin{array}{l}\text { - Resultados visibles en menor } \\
\text { tiempo } \\
\text { - Disminución de la depresión y la } \\
\text { ansiedad de las personas con EA }\end{array}$ & • Tamaño muestral pequeño \\
\hline $\begin{array}{l}\text { Mowrey et } \\
\text { al."1 (2012) }\end{array}$ & $\begin{array}{l}\text { Determinar la viabilidad práctica y la efectividad de } \\
\text { la intervención ergonómica que se realizó en una } \\
\text { unidad de demencia en una residencia de atención } \\
\text { continua }\end{array}$ & Estudio piloto & $N=39$ & $\begin{array}{l}\text { - Disminución de depresión, } \\
\text { ansiedad y agitación en residentes } \\
\text { - Mejor calidad de vida de los } \\
\text { residentes con EA en la reducción } \\
\text { de caídas, el estado de ánimo y la } \\
\text { cantidad de cambios conductuales }\end{array}$ & - Estudio retrospectivo \\
\hline $\begin{array}{l}\text { Keshavarz et } \\
\text { al. }^{12}(2017)\end{array}$ & $\begin{array}{l}\text { Determinar los efectos del masaje de cabeza y } \\
\text { cara sobre la agitación en pacientes ancianos con } \\
\text { Alzheimer que viven en residencias de ancianos }\end{array}$ & $\begin{array}{l}\text { Ensayo clínico } \\
\text { aleatorizado } \\
\text { y controlado }\end{array}$ & $N=70$ & $\begin{array}{l}\text { - Reducción de la agitación en } \\
\text { pacientes ancianos con Alzheimer }\end{array}$ & $\begin{array}{l}\text { - Discrepancia en los resultados por } \\
\text { diferencias en la cantidad de centros } \\
\text { de atención, el tipo de masaje y la } \\
\text { cantidad de sesiones de masaje }\end{array}$ \\
\hline $\begin{array}{l}\text { Bharwani et } \\
\text { al. }^{13}(2012)\end{array}$ & $\begin{array}{l}\text { Reducir el estrés cognitivo y los problemas de } \\
\text { comportamiento (o relacionados con el estado de } \\
\text { ánimo) como la agitación con el uso de actividades } \\
\text { ergonómicas }\end{array}$ & Estudio piloto & $N=18$ & $\begin{array}{l}\text { - Prevención de episodios } \\
\text { posteriores de agitación }\end{array}$ & $\begin{array}{l}\text { - Requiere de personal cualificado, } \\
\text { lo que limita el número de veces } \\
\text { que puede realizarse } \\
\text { - Tamaño muestral pequeño }\end{array}$ \\
\hline $\begin{array}{l}\text { Resnick et } \\
\text { al. }^{14}(2017)\end{array}$ & $\begin{array}{l}\text { Determinar si la población expuesta al TIE } \\
\text { demuestra evidencia de implementación evaluada } \\
\text { por los criterios de alcance, efectividad, adopción, } \\
\text { implementación y mantenimiento } \\
\text { Evaluar la viabilidad, utilidad y costo del TIE }\end{array}$ & $\begin{array}{l}\text { Protocolo de } \\
\text { investigación }\end{array}$ & $N=625$ & $\begin{array}{l}\text { - Prevención y manejo de cambios } \\
\text { conductuales en entornos de } \\
\text { atención a largo plazo }\end{array}$ & $\begin{array}{l}\text { - Enfoque limitado en la difusión o } \\
\text { implementación de hallazgos en } \\
\text { contextos reales }\end{array}$ \\
\hline $\begin{array}{l}\text { Chen et al. }{ }^{15} \\
(2019)\end{array}$ & $\begin{array}{l}\text { Investigar si el ejercicio pudiera usarse para } \\
\text { mejorar la alimentación en pacientes con } \\
\text { Alzheimer }\end{array}$ & $\begin{array}{l}\text { Estudio } \\
\text { experimental }\end{array}$ & $N=60$ & $\begin{array}{l}\text { - Intervención segura y efectiva } \\
\text { para mejorar la alimentación } \\
\text { de las personas con EA }\end{array}$ & $\begin{array}{l}\text { - Muestra pequeña y poco tiempo } \\
\text { de evaluación } \\
\text { - No generalización estadística }\end{array}$ \\
\hline $\begin{array}{l}\text { Cantarella et } \\
\text { al. }{ }^{16}(2017)\end{array}$ & $\begin{array}{l}\text { Medir el impacto de la terapia con muñecas en } \\
\text { personas con demencia con una escala confiable } \\
\text { y de uso común para evaluar los cambios } \\
\text { conductuales y la angustia relacionada en los } \\
\text { cuidadores }\end{array}$ & $\begin{array}{l}\text { Estudio } \\
\text { experimental }\end{array}$ & $N=29$ & $\begin{array}{l}\text { - Reducción de los cambios } \\
\text { conductuales en las personas } \\
\text { conEA }\end{array}$ & $\begin{array}{l}\text { - Falta de seguimiento a largo plazo } \\
\text { - Tamaño muestral pequeño } \\
\text { - No generalización de resultados }\end{array}$ \\
\hline
\end{tabular}

EA: enfermedad de Alzheimer; OR: orientación a la realidad; TIE: triángulo de integración de la evidencia; TR: terapia de reminiscencia.

Tabla 2. Intervenciones de enfermería a pacientes con enfermedad de Alzheimer en fase moderada

\begin{tabular}{|c|c|c|c|c|c|c|c|c|}
\hline \multirow[b]{2}{*}{ Tіро } & \multirow{2}{*}{$\begin{array}{l}\text { Intervenciones } \\
\text { de enfermería }\end{array}$} & \multicolumn{7}{|c|}{ Estudios } \\
\hline & & $\begin{array}{l}\text { Onieva et al. }{ }^{10} \\
\text { (2018) }\end{array}$ & $\begin{array}{l}\text { Mowrey et al.11 } \\
\text { (2012) }\end{array}$ & $\begin{array}{c}\text { Keshavarz et al. }{ }^{12} \\
\text { (2017) }\end{array}$ & $\begin{array}{c}\text { Bharwani et al. }{ }^{13} \\
\text { (2012) }\end{array}$ & $\begin{array}{l}\text { Resnick et al. }{ }^{14} \\
\text { (2017) }\end{array}$ & $\begin{array}{l}\text { Chen et al. }{ }^{15} \\
\text { (2019) }\end{array}$ & $\begin{array}{c}\text { Cantarella et al. }{ }^{16} \\
\text { (2017) }\end{array}$ \\
\hline \multirow{4}{*}{ Generales } & $\begin{array}{l}\text { Intervenciones sensitivas } \\
\text { (musicoterapia, masajes } \\
\text { manuales) }\end{array}$ & • & - & - & & & & \\
\hline & Intervenciones ambientales & & & & - & $\bullet$ & & \\
\hline & $\begin{array}{l}\text { Intervenciones físicas } \\
\text { (ejercicio) }\end{array}$ & & & & & & - & \\
\hline & $\begin{array}{l}\text { Intervenciones } \\
\text { psicoemocionales (terapia } \\
\text { con muñecos, caja con } \\
\text { artículos de apoyo) }\end{array}$ & & - & & • & & & - \\
\hline \multicolumn{2}{|c|}{ Integran a la familia } & & $\bullet$ & - & - & & - & - \\
\hline
\end{tabular}


al ser fácil de ejecutar puede ser aplicada tanto por los profesionales de enfermería como por los familiares, lo que fomenta un encuentro agradable entre la paciente y su familia, fortaleciendo así su relación. Por todo lo argumentado previamente, esta intervención podría ser una buena opción para este caso, ya que la familia estaba presente la mayor parte del tiempo, pero no sabía cómo manejar la enfermedad, lo que, en ocasiones, como se objetivó, podía perjudicar tanto a la paciente como a la propia familia.

\section{Intervenciones ambientales}

La terapia TEBC también incorpora la utilización de intervenciones ambientales en su programa, las cuales consisten en la modificación del entorno físico o social, mediante, por ejemplo, la eliminación de restricciones físicas y el contacto social real, con el fin de reducir el estrés y los cambios conductuales ${ }^{13}$. En el caso descrito, también se considera importante favorecer un ambiente en el que la paciente se sienta segura para evitar que su comportamiento se altere. En el momento en que la residente manifestó dichos cambios, en forma de agitación, gritos y violencia, el entorno en el que se encontraba estaba caracterizado por nerviosismo, ruido y presión, lo que pudo promover un entorno inseguro. Otra intervención sugerida es el uso del triángulo de integración de la evidencia (TIE), que consta de tres elementos: el proceso de implementación participativa, la aplicación de enfoques basados en la evidencia y medidas prácticas de progreso ${ }^{14}$. Según Chambers et al., esta terapia tiene un impacto potencial significativo en los pacientes con EA institucionalizados en los centros residenciales, ya que proporciona una orientación sobre cómo tratar los cambios conductuales y psicológicos ${ }^{14}$. Esta consiste en 4 pasos:

1. Evaluación del medio ambiente y las políticas para la aplicación de intervenciones en los cambios conductuales.

2. La educación del personal sobre los cambios conductuales.

3. Establecimiento de planes de atención centrada en la persona desarrollados por el personal.

4. Motivación del equipo para integrar enfoques conductuales centrados en la persona de manera rutinaria ${ }^{14}$.

Esta intervención puede ayudar en gran medida en el caso, ya que asocia la importancia del ambiente, el cual estaba alterado, con la educación y motivación del personal sobre los cuidados y los cambios conductuales. En concreto, la promoción de un ambiente seguro, junto con la educación del personal de nueva incorporación sobre los cambios conductuales derivados de la enfermedad y la elaboración de un plan de atención centrado en la persona, así como su motivación, podría haber prevenido el cambio conductual de la residente. Esto es así porque la falta de conocimientos y de motivación por parte de la enfermera pudo dificultar que se estableciera una relación de confianza con la residente.

\section{Intervenciones físicas}

El ejercicio físico es la principal intervención física que se realiza a las personas con EA. Esto es debido a sus múltiples beneficios sobre su función cognitiva; por ejemplo, en la memoria o en la capacidad de atención de estos pacientes ${ }^{15}$. Esta intervención, aunque generalmente es llevada a cabo por el personal de enfermería, tal y como sugieren Chen et al. ${ }^{15}$, podría integrar también a los familiares. De este modo, mejoraría la relación entre los pacientes y profesionales, al incrementar su presencialidad y mejorar su respuesta ante sus necesidades, pudiendo entender mejor los hábitos y el significado de sus comportamientos y, en consecuencia, proporcionar una atención más adecuada ${ }^{15}$. A este respecto, esta actividad podría ser beneficiosa en este caso.

\section{Intervenciones psicoemocionales}

Entre las intervenciones psicoemocionales destaca la terapia con muñecas, sobre todo en mujeres con EA. Uno de los objetivos del estudio de Cantarella et al. ${ }^{16}$ fue determinar la eficacia de esta en la reducción de la frecuencia e intensidad de los cambios conductuales en personas con EA. El efecto que crea es una reactivación de experiencias o actitudes previas de maternidad, y actúa a nivel emocional, reduciendo la apatía. Esta terapia ayuda a las personas con demencia a encontrar alivio, satisfacer sus necesidades de apego y, en consecuencia, calmar sus cambios conductuales. También puede ser una manera de contener el sentido de soledad ${ }^{16}$. Para la paciente del presente caso parece conveniente utilizarla, con el fin de favorecer que su actitud sea más relajada y emocional, tanto con la familia como con el equipo profesional.

El programa TEBC incluye también en su práctica una caja de apoyo, en la que se almacenan artículos sentimentales proporcionados por los miembros de la familia. Estos artículos pueden ser fotos familiares, revistas, libros o animales de peluche, entre otros. La visualización de estos artículos ayuda a mitigar la depresión, ansiedad y agitación de los pacientes con EA, lo que conlleva una mejora de su calidad de vida ${ }^{11,13}$. Este tipo de intervención podría ser útil en este caso ya que, al fomentar un cambio en su estado de ánimo, pudo ayudar a prevenir los cambios conductuales que experimentó a la hora de movilizarla. Por otro lado, integra a la familia al implicarla en la obtención de los materiales requeridos, como las fotografías, vídeos y objetos sentimentales, favoreciendo un mayor vínculo, dado que ayuda tanto a la paciente como a sus familiares a rememorar vivencias pasadas.

IMPACTO DE LA INTEGRACIÓN DE LA FAMILIA EN EL CUIDADO DEL PACIENTE CON ENFERMEDAD DE ALZHEIMER

La integración de la unidad familiar en el cuidado del paciente con EA es clave por sus beneficios tanto para la salud de los residentes como de la propia familia. No obstante, cabe señalar la escasez de intervenciones desarrolladas en centros geriátricos que integren a la familia en el cuidado de los pacientes. Entre estas intervenciones, detalladas en la anterior sección, se encuentran la terapia con masajes, el ejercicio físico, la terapia con muñecas y la caja de apoyo ${ }^{11-13,15,16}$.

Los cambios conductuales que padecen los pacientes con EA en fase moderada tienen un impacto en la propia salud de los familiares, afectando a su confianza y bienestar e incrementando su estrés ${ }^{13}$. Por ello, es conveniente que las intervenciones para manejar los cambios conductuales se realicen cuando los miembros de la familia están visitando al residente, lo que permite mejorar sus experiencias con sus seres queridos ${ }^{11}$.

La integración de los familiares en el manejo y prevención de los cambios conductuales favorece que los pacientes se sientan acompañados ${ }^{14}$. En este sentido, por ejemplo, Keshavarz et al. ${ }^{12}$ sugieren la importancia de capacitar a los miembros de la familia en la realización de los masajes como tratamiento eficaz. Por su parte, Cantarella et al. ${ }^{16}$ recomiendan la terapia con muñecas para mejorar el bienestar y la calidad de vida de los cuidadores principales. En el programa TEBC se implica a la familia para aportar los materiales necesarios, como vídeos familiares, fotografías y música, para cada paciente ${ }^{13}$. Otra buena opción de integración familiar sería el diseño de un curso de capacitación de los cuidadores principales para la atención de sus familiares con $\mathrm{EA}^{15}$.

En el caso descrito, la paciente buscaba ayuda en su familia cuando la intentaban movilizar entre todos, pero, al percibir que se enfrentaban también a ella, sus cambios conductuales se acentuaron. Dado que la familia acompaña a la paciente la mayor parte del día, se cree conveniente que conozca los cuidados que precisa para el manejo de los posibles cambios conductuales, para garantizar una mayor efectividad 
de estos. En concreto, se podría capacitar e integrar a la familia en la implementación de la terapia con masajes, el ejercicio físico, la terapia con muńecas y la caja de apoyo para esta paciente. En este sentido, se considera relevante evaluar el impacto de la integración de la familia en el cuidado de esta paciente con EA en fase moderada durante su institucionalización.

\section{- CONCLUSIONES}

A través de este trabajo se ha analizado un caso clínico, basándose en la literatura científica, de una paciente con EA en fase moderada durante su proceso de institucionalización en un centro gerontológico, teniendo en cuenta la integración de su familia. Con base en los hallazgos existentes en torno a este fenómeno, se proponen distintas intervenciones de enfermería, sensitivas, ambientales, físicas y psicoemocionales, que podrían beneficiar tanto a la residente y familia del caso como al personal de enfermería.

Cabe señalar, sin embargo, que estas intervenciones deben tratarse con cautela dadas las limitaciones metodológicas de los estudios identificados (véase tabla 1). En este sentido, y dada su escasez, sería conveniente llevar a cabo futuros estudios de tipo intervención que integren a la familia, incluyan un muestreo aleatorio, un tamaño muestral suficiente para poder generalizar los resultados, y un seguimiento a largo plazo.
Siendo conscientes de estas limitaciones, la integración de la familia en el cuidado de la paciente de este caso se considera clave, dado su potencial impacto sobre la residente, la enfermera y familia, en tanto que ayudaría a manejar y prevenir los cambios conductuales, al disminuir su estado de agitación, agresión y depresión, mejoraría el bienestar de los familiares y reduciría su estrés. Así mismo, los profesionales de enfermería tienen un papel fundamental en este caso, ya que son el nexo de unión entre la paciente y su familia, encontrándose en una posición privilegiada para implementar y evaluar las intervenciones planteadas. En esta línea, no se debe obviar la importancia que, para llevar a cabo este tipo de intervenciones, tiene el favorecer e impulsar la formación de los profesionales en la atención a los pacientes con EA en fase moderada y a su familia.

Entre las limitaciones de este trabajo es necesario señalar que los resultados obtenidos responden a la búsqueda de las principales bases de datos, habiéndose acotado a los diez últimos años y al idioma de publicación, por lo que puede haberse omitido algún estudio de interés para el tema abordado. No obstante, cabe mencionar que los hallazgos identificados han ayudado a analizar el caso propuesto y que, a través de la revisión realizada, se han identificado vacíos que precisan ser cubiertos

\section{Conflicto de intereses}

Se declara que no existe conflicto de intereses por parte de los autores con el artículo publicado.

\section{- BIBLIOGRAFÍA}

1. Organización Mundial de la Salud, 2019. Disponible en: https:// www.who.int/es/news-room/fact-sheets/detail/dementia

2. Patterson C. Informe mundial sobre el Alzheimer. La investigación de vanguardia sobre la demencia: Nuevas fronteras. Londres: Alzheimer's Disease International (ADI); 2018 [fecha de consulta 14 de febrero de 2020]. Disponible en: https://www.alz.co.uk/research/worldalzheimerreport2018-spanish.pdf

3. Instituto Nacional de Estadística [Internet] 2018. [fecha de consulta 20 de febrero de 2020]. Disponible en: https://www.ine.es/prodyser/espa_cifras/2018/files/assets/common/downloads/publication.pdf?uni=4f7e7b429c56ccbc4bf56b3e93ebc47b

4. Castellón Sánchez del Pino A, Gómez Arques MA, Martos Martín A. Alteraciones conductuales en la enfermedad de Alzheimer. Semergen. 2005:31(11):541-5. https://doi.org/10.1016/s1138-3593(05)72987-7

5. Regier NG, Hodgson NA, Laura N Gitlin LN. Characteristics of Activities for Persons With Dementia at the Mild, Moderate, and Severe Stages. The Gerontologist. 2017;57(5):987-97. https://doi. org/10.1093/geront/gnw133

6. Kales HC, Lyketsos CG, Miller EM, Ballard C. Management of behavioral and psychological symptoms in people with Alzheimer's disease: an international Delphi consensus. Int Psychogeriat 2019;31(1):83-90. doi:10.1017/S1041610218000534
7. Liaño Balbas VM. El Profesional de Enfermería y el Alzheimer NURE Investigación. 2015;2(13). Disponible en: http://www.nure. org/OJS/index.php/nure/article/view/223

8. Esandi Larramendi N, Canga-Armayor A. Familia cuidadora y enfermedad de Alzheimer: una revisión bibliográfica. Gerokomos. 2011;22(2):56-61. https://doi.org/10.4321/s1134-928x2011000200002

9. Huang HL, Shyu YI, Chen MC, Huang C-C. Family caregivers' role implementation at different stages of dementia. Clin Interv Aging. 2015;10:135-46. doi:10.2147/CIA.S60574

10. Onieva-Zafra MD, Hernández-Garcia L, Gonzalez-Del-Valle MT, Parra-Fernández ML, Fernandez-Martinez E. Music Intervention with Reminiscence Therapy and Reality Orientation for Elderly People with Alzheimer Disease Living in a Nursing Home: A Pilot Study. Holist Nurs Pract. 2018;32(1):43-50. https://doi.org/10.1097/ HNP.0000000000000247

11. Mowrey C, Parikh PJ, Bharwani G, Bharwani M. Application of behavior-based ergonomics therapies to improve quality of life and reduce medication usage for Alzheimer's/dementia residents. Am J Alzheimers Dis Other Demen. 2013;28(1):35-41. https://doi. org/10.1177/1533317512467678

12. Keshavarz S, Mirzaei T, Ravari A. Effect of head and face massage on agitation in elderly Alzheimer's disease patients. Evidence
Based Care Journal. 2018;7(4):46-54. https://doi.org/10.22038/ ebcj.2017.25958.1589

13. Bharwani G, Parikh PJ, Lawhorne LW, Vanvlymen E, Bharwani M Individualized Behavior Management Program for Alzheimer's/ Dementia Residents Using Behavior-Based Ergonomic Therapies. Am J Alzheimers Dis Other Demen. 2012;27(3):188-95. https://doi. org/10.1177/1533317512443869

14. Resnick B, Kolanowski A, Van Haitsma K, Galik E, Boltz M, Ellis JZ et al. Testing the evidence integration triangle for implementation of interventions to manage behavioral and psychological symptoms associated with dementia: Protocol for a pragmatic trial. Res Nurs Health. 2018;41(3):228-42. https://doi.org/10.1002/nur.21866

15. Chen LL, Li H, Chen XH, Jin S, Chen OH, Chen MR, Li N. Effects of Hand Exercise on Eating Action in Patients With Alzheimer's Disease. Am J Alzheimers Dis Other Demen. 2019;34(1):57-62. https:// doi.org/10.1177/1533317518803722

16. Cantarella, A, Borella, E, Faggian S, Navuzzi A, De Beni R. Using dolls for therapeutic purposes: A study on nursing home residents with severe dementia. Int J Geriatr Psychiatry. 2018 Jul;33(7):91525. https://doi.org/10.1002/gps.4872 